\title{
Pretransitional Phenomena of a Colloid Polymer Mixture Studied with Static and Dynamic Light Scattering
}

\author{
Igor Bodnár,* Jan K. G. Dhont, and Henk N. W. Lekkerkerker \\ Van't Hoff Laboratory, Utrecht University, Padualaan 8, 3584 CH, Utrecht, The Netherlands
}

Received: August 19, $1996^{\otimes}$

\begin{abstract}
A mixture of hard-sphere colloidal silica particles (radius $48 \mathrm{~nm}$ ) and a nonadsorbing polymer (poly(dimethylsiloxane), radius of gyration $23 \mathrm{~nm}$ ) is studied by means of static and dynamic light scattering near the binodal. The spinodal is determined from an extrapolation of the diffusion coefficient as measured in the one-phase region, where it is essential to take the hydrodynamic interactions into account. The distance between the binodal and spinodal is small in the entire region so that it is difficult to locate the critical point accurately. The correlation length, measured with static light scattering, increases drastically on approaching the binodal. From these measurements the spinodal could be determined as well. For not yet understood reasons, there is a considerable discrepancy between the location of the spinodal as found from extrapolated dynamic and static light scattering data.
\end{abstract}

\section{Introduction}

Attractive interactions between hard-sphere colloidal particles can be induced by adding nonadsorbing polymer. ${ }^{1,2}$ This depletion-induced attraction can be tuned by the concentration and the size of the polymer. On increasing the attractive interactions, a phase separation will occur. The type of phase separation depends on the size ratio of the colloidal particles and the polymer. ${ }^{3-10}$ Our interest in this article is focused on the experimental determination of the location of phase lines, relating to the gas-liquid transition (dilute and concentrated disordered phases).

Light scattering studies, probing pretransitional phenomena, are performed to locate the spinodal. Although the spinodal cannot be measured directly, due to the existence of a metastable region between the binodal and spinodal, it is possible to measure quantities that change significantly in the stable region of the phase diagram due to the presence of the spinodal. The diffusion coefficient, for instance, vanishes at the spinodal. In the unstable region of the phase diagram, this diffusion coefficient is negative, giving rise to spinodal decomposition. This lowering of the diffusion coefficient in the vicinity of the spinodal is usually referred to as critical slowing down. In addition to a vanishing of the diffusion coefficient, the correlation length diverges at the spinodal, giving rise to critical opalescence. The correlation length is directly related to the small angle scattered intensity. ${ }^{11}$

Phase separation into a dilute and a dense disordered colloidal phase is also found in adhesive hard-sphere suspensions. In addition, in adhesive hard-sphere suspensions a gel-line is found, ${ }^{12,13}$ which seems to intersect with the critical point. ${ }^{13}$ Such a gel-line is not observed in colloid polymer mixtures studied here, making the critical region better accessible for the study of critical phenomena. An essential difference between the two types of systems is that in colloid polymer mixtures the concentration of polymer sets the strength of the attractions, contrary to the adhesive hard-sphere system discussed in ref 13, where the temperature is the relevant variable. The polymer concentration plays qualitatively the role of an inverse temperature: lowering the concentration of the polymer reduces the magnitude of the attraction, which is otherwise accomplished by increasing the temperature.

${ }^{\otimes}$ Abstract published in Advance ACS Abstracts, November 1, 1996.
The aim of this paper is to determine the location of the binodal and spinodal by means of visual observation and light scattering experiments, respectively. Static and dynamic light scattering experiments are performed in the stable part of the phase diagram, and data are suitably extrapolated to obtain the spinodal.

In section II a short overview of the theory is given according to which the data are analyzed. It turns out that, for colloidal systems, long-ranged hydrodynamic interactions affect the behavior of the diffusion coefficient close to the spinodal in an essential way. In section III the system and the experimental setup are described. In section IV the results of direct visual observation and static and dynamic light scattering are given. Finally in section $\mathrm{V}$ we end with some concluding remarks.

\section{Theory}

A. Static Light Scattering. The intensity of light scattered by a monodisperse suspension is given by

$$
I(k) \approx P(k) S(k)
$$

where $P(k)$ is the form factor, which depends on the optical properties of the individual particles, and $S(k)$ is the structure factor, which measures the microstructural order. Furthermore, $k$ is the wave vector, which is related to the scattering angle $\theta$ as

$$
k=\frac{4 \pi}{\lambda} \sin \left(\frac{\theta}{2}\right)
$$

with $\lambda$ the wavelength of the light in the suspension.

The structure factor is essentially the Fourier transform of the pair-distribution function and contains information about the microstructure of the dispersion. Close to the spinodal, large-scale microstructures exist, giving rise to large scattered intensities at small scattering angles. The size of these structures is characterized by the correlation length $\xi$, which is proportional to the square root of the osmotic compressibility $\partial \rho / \partial \Pi$,

$$
\xi=\sqrt{\frac{\Sigma}{\partial \Pi / \partial \rho}}
$$

which becomes infinite at the spinodal since there $\partial \Pi / \partial \rho=0$. 
The quantity $\Sigma$ is related to the Cahn-Hilliard square gradient coefficient, ${ }^{14}$ which is well behaved in the vicinity of the spinodal. To determine the correlation length from scattering curves, the Ornstein-Zernike approximation is used for the structure factor,

$$
S(k)=(\beta \Sigma)^{-1} \frac{1}{\xi^{-2}+k^{2}}
$$

This approximation, which is valid near the spinodal, neglects terms of order $k^{4}$ and higher. The correlation length is experimentally found by measuring the wave vector dependence of the scattered intensity and taking the square root of the ratio of the intercept and the slope of plots of the reciprocal scattered intensity against $k^{2}$. The spinodal can then be found by extrapolating the correlation length to infinity or, equivalently, the reciprocal correlation length to zero.

B. Dynamic Light Scattering. With dynamic light scattering the intensity autocorrelation function is measured. For monodisperse suspensions and short times, the following expression holds for the normalized correlation function $\hat{g}_{I}(k, t)$,

$$
\hat{g}_{I}(k, t)=1+\mathrm{e}^{-2 D(k) k^{2} t}
$$

where $D$ is the short time collective diffusion coefficient. For slightly polydisperse systems a small quadratic term in time must be added to the argument of the exponential function.

$$
\hat{g}_{I}(k, t)=1+\mathrm{e}^{-2 D(k) k^{2} t+E(k)_{t}^{2}}
$$

The diffusive behavior of a system changes dramatically when the spinodal is approached: the short time collective diffusion coefficient $D$ becomes zero on the spinodal, for zero wave vector. After crossing the spinodal the diffusion coefficient becomes negative for small wave vectors, giving rise to spinodal decomposition. The following expression gives $D$ in terms of the static structure factor $S(k)$ and the hydrodynamic mobility function $H(k):^{15,16}$

$$
D=D_{0} \frac{H(k)}{S(k)}
$$

where $D_{0}$ is the Stokes-Einstein diffusion coefficient. The static structure factor is given in eq 4 , and the hydrodynamic mobility function is given by

$$
H(k)=\frac{1}{N} \sum_{i, j=1}^{N}\left\langle\left(\hat{\mathbf{k}} \cdot \frac{\mathbf{D}_{i j}}{D_{0}} \cdot \hat{\mathbf{k}}\right) \mathrm{e}^{i \mathbf{k} \cdot\left(\mathbf{r}_{\mathrm{i}}-\mathbf{r}_{\mathrm{j}}\right)}\right\rangle
$$

which is an ensemble average involving hydrodynamic interaction matrices $\boldsymbol{D}_{i j} .{ }_{15,16}$ Close to the spinodal this ensemble average is essentially an integral weighted by the total correlation function $h(r)$, given by

$$
h(r)=\frac{1}{\beta \Sigma} \frac{1}{4 \pi \rho} \frac{\mathrm{e}^{-\mathrm{r} / \xi}}{r}
$$

Without hydrodynamic interactions, the matrices $\boldsymbol{D}_{i j}$ are simply given by

$$
\mathbf{D}_{i j}=D_{0} \hat{\mathbf{I}} \delta_{i j}
$$

in which $\delta_{i j}$ is the Kronecker delta. Substituting this into eq 8, it follows that $H(k)=1$. Substitution of eq 4 for the static structure factor together with eq 3 for the correlation length into eq 7 then yields

$$
D=D_{0} \beta\left(\frac{\partial \Pi}{\partial \rho}+\Sigma k^{2}\right)=D_{0}(\beta \Sigma)\left(\xi^{-2}+k^{2}\right)
$$

Close to the spinodal, where direct interactions become longranged, hydrodynamic interactions must be included, giving rise to an extra term for the diffusion coefficient, which will become dominant near the spinodal.

Due to the long-range character of the Oseen contribution to the hydrodynamic interaction matrices, the hydrodynamic mobility function $H(k)$, at zero wave vector, diverges on the spinodal. Higher order hydrodynamic interactions than the Oseen contribution give rise to finite contributions to $H(k)$ and need not be considered here. Up to the Oseen level, the diagonal hydrodynamic interaction matrices are equal to

$$
\mathbf{D}_{i i}=D_{0} \hat{\mathbf{I}}
$$

and the off-diagonal terms are given by

$$
\mathbf{D}_{i j}=D_{0} 6 \pi \eta_{0} a \mathbf{T}(\mathbf{r}), \quad i \neq j
$$

where $a$ is the radius of the particle, $\eta_{0}$ the viscosity of the solvent, and $\mathbf{T}(\mathbf{r})$ the Oseen matrix

$$
\mathbf{T}(\mathbf{r})=\frac{1}{8 \pi \eta_{0}} \frac{1}{r}[\hat{\mathbf{I}}+\hat{\mathbf{r}} \hat{\mathbf{r}}]
$$

with $\hat{\mathbf{r}}=\mathbf{r} / r$.

Substitution of eqs $9,12,13$, and 14 into eq 8 for $H(k)$ leads to the following expression for the diffusion coefficient, which is essentially eq 11 with an extra term due to the hydrodynamic interactions: ${ }^{14}$

$$
D=D_{0}\left[(\beta \Sigma)\left(\xi^{-2}+k^{2}\right)+\frac{a}{\xi}(k \xi)^{-2} \mathrm{~F} \quad(k \xi)\right]
$$

with $F$ the Kawasaki function,

$$
\mathrm{F}(z)=\frac{3}{4}\left[1+z^{2}+\left(z^{3}-z^{-1}\right) \arctan (z)\right]
$$

This function was first encountered by Kawasaki and rederived by Ferrell in their seminal studies on critical phenomena in fluids. ${ }^{17,18}$ Notice that we find that for colloidal systems the Kawasaki function appears as an additive term instead of a multiplicative factor as in fluids.

Expanding eq 15 in a Taylor series in $1 / k \xi$ for large $k \xi$ yields

$$
\begin{aligned}
D & =D_{0}\left[\frac{\beta \Sigma}{\xi^{2}}(k \xi)^{2}+\frac{3 \pi}{8} \frac{a}{\xi}(k \xi)+\frac{\beta \Sigma}{\xi^{2}}+\frac{a}{\xi} \frac{1}{(k \xi)^{2}}+\frac{a}{\xi} 0\left(\frac{1}{(k \xi)^{3}}\right)\right] \\
& =D_{0}\left[\frac{\beta \Sigma}{a^{2}}(k a)^{2}+\frac{3 \pi}{8}(k a)+\frac{\beta \Sigma}{\xi^{2}}+\frac{a}{\xi} \frac{1}{(k \xi)^{2}}+\frac{a}{\xi} 0\left(\frac{1}{(k \xi)^{3}}\right)\right]
\end{aligned}
$$

Notice that the first two terms are independent of $\xi$, so that for large $k \xi$ the $k$ dependence of $D$ is independent of the distance to the spinodal, except that curves of $D$ versus $k$ have a different "offset" equal to $D_{0} \beta \Sigma / \xi^{2}$. The form of the $k$ dependence of $D$ (curvature and slope) are independent of $\xi$, but $D$ is displaced by a constant $D_{0} \beta \Sigma / \xi^{2}$, which depends strongly on the distance to the spinodal. Hence, on approach of the spinodal, curves of $D$ versus $k$ all have the same form and are displaced relative to each other, depending on the correlation length.

Note that the first term in eq $17, \beta \Sigma k^{2}$, is much larger than the offset term, $\beta \Sigma / \xi^{2}$, for large values of $k \xi$. The former term determines the correlation length independent shape of the curves of $D$ versus $k$, whereas the latter sets the correlation 
length dependent offset. This is why, on varying the distance to the spinodal, the term $\beta \Sigma / \xi^{2}$ cannot be neglected: this term is the only term that is responsible for a change of $D$ versus $k$ curves on approach of the spinodal.

A crude estimate of the dimensionless number $\beta \Sigma / R_{V}^{2}$ is $1 / 10 .{ }^{19,20}$ Using this value with $R_{V} \approx 2 a$, one finds from eq 17 , for large $k \xi$, that the first term, $\left(\beta \Sigma / a^{2}\right)(k a)^{2}$, is less than $10 \%$ of the second term, $(3 \pi / 8)(k a)$, for $k a<1 / 4$. In this wave vector range, $D$ versus $k$ is then expected to be approximately a linear function of $k$ with a correlation length independent slope. Linear extrapolation of $D$ versus $k$ to $k=0$ then yields $D(k=0)$ $=D_{0} \beta \Sigma / \xi^{2}$.

\section{Experimental Methods}

A. The System. The system under study consists of sterically stabilized stearylsilica, synthesized according to refs 21-23. The particles have an average radius of $48 \mathrm{~nm}$, determined with dynamic light scattering. With transmission electron microscopy a relative polydispersity of $10 \%$ is found. The shape of the particles is not perfectly spherical, but there is no significant anisotropy. The system is dispersed in cyclohexane, where the interaction potential resembles a hardsphere potential.

The polymer used is poly(dimethylsiloxane) (PDMS, Janssen) with a molecular weight of $204 \mathrm{~K}$ and a radius of gyration in cyclohexane of $23 \mathrm{~nm}$ at $25^{\circ} \mathrm{C}$.

With this size ratio of polymer/colloid of 0.48 a gas-liquid phase transition is expected as well as a gas-solid phase transition, on the basis of the theoretical predictions of Lekkerkerker et al. ${ }^{6}$

B. Binodal. The binodal was determined by visual observation. This was done in the following way. A suspension with fixed amounts of colloid and polymer is concentrated by slowly evaporating cyclohexane under a nitrogen flow. A suspension is diluted by simply adding cyclohexane. In this way a socalled dilution line in the phase diagram with a constant colloid/ polymer concentration ratio is probed. For each dilution line the binodal is determined as the middle between the last point where the equilibrated system is still homogeneous and the first point where phase separation is observed within a few hours. This phase boundary is crossed several times, by dilution and concentration, in order to determine the binodal to within the desired accuracy. The concentrations were calculated from the known amounts of colloid and polymer in the sample and the total weight of the sample.

After a subsequent series of light scattering measurements along a single dilution line, some colloid was added to the sample, resulting in a system with a different colloid/polymer concentration ratio. Along this new dilution line the binodal was determined again and a new series of measurements were performed. Relatively large amounts of colloid and polymer were used, in order to minimize the error due to evaporation.

C. Spinodal. 1. Static Light Scattering. The static light scattering setup consists of a $5 \mathrm{~mW} \mathrm{He}-\mathrm{Ne}$ laser (wavelength $638.2 \mathrm{~nm}$ ), of which the intensity is controlled by attenuating the primary beam with two polarizers. Two mirrors are used for ease of the alignment. A pinhole and a diaphragm are used in order to be able to vary the scattering volume in the cuvette and to prevent other than the laser light from entering the light scattering apparatus, which itself is enclosed in a light-tight box. The cuvette is placed in a container filled with toluene. This container serves three purposes. (i) It serves as a thermostat. A cylindrical glass ring of optical quality with a beam inlet is clamped between two metal elements through which the thermostating water flows. The connection between the metal elements and the glass ring is established via two Teflon rings to prevent leakage of toluene. (ii) It is filled with toluene that matches the refractive index of the glass of the cuvette so as to avoid spurious scattering from optical imperfections of the cuvette. Inside the toluene bath there is a beamguide between the beam inlet and the cuvette, to prevent the detection of any stray light scattered from the inlet. (iii) It serves as a lens for the scattered light: the radius of the glass ring is such that the scattered intensity at any scattering angle is focused onto the detector at a distance of $179 \mathrm{~mm}$ from the cuvette.

The detector is a linear diode array camera, model M1452A of EG\&G, where each of the 512 diodes has the dimensions $2.5 \mathrm{~mm} \times 25 \mu \mathrm{m}$. The time resolution of this camera is 10 $\mathrm{ms}$, its dynamic range is $2^{14}$, and its sensitivity $\approx 1$ per 2800 photons at a wavelength of $550 \mathrm{~nm}$. The camera is cooled to $5{ }^{\circ} \mathrm{C}$. To prevent condensation it is flushed with dry nitrogen. The detector can be moved around the toluene bath in order to select the desired scattering angle range.

The static light scattering curves were measured at $25^{\circ} \mathrm{C}$. The samples were allowed to equilibrate for about $15-50 \mathrm{~min}$. The scattered intensity was measured in the scattering angle range $1.87^{\circ}-5.95^{\circ}$ in steps of $0.008^{\circ}$. The samples were contained in a rectangular quartz cuvette of $1 \mathrm{~cm} \times 1 \mathrm{~cm}$. Within a series of measurements along a single dilution line the intensity of the primary beam was kept constant, so that also the various scattered intensities can be compared.

2. Dynamic Light Scattering. Dynamic light scattering (dls) measurements were performed in a similar setup as described above for static light scattering. The detector is a photomultiplier tube connected with a Malvern $7032 \mathrm{CE}$ correlator.

The dls measurements were done in a cylindrical glass tube with a diameter of about $2 \mathrm{~cm}$. A laser with a wavelength of $514.5 \mathrm{~nm}$ was used. The samples were measured at $25^{\circ} \mathrm{C}$ at angles between $20^{\circ}-50^{\circ}$ with steps of $5^{\circ}$. Each sample was allowed to equilibrate for $15-60 \mathrm{~min}$.

\section{Results and Discussion}

A. Binodal. The time required to observe phase separation varies from several minutes to hours, depending on the colloid/ polymer concentration ratio. When the volume of the separated phases are almost equal, the phase separation time increases, indicating so-called critical slowing down. This is observed in the region where the volume fraction of colloid, $\phi_{\mathrm{c}}$, is between $19 \%$ and $23 \%$. This is the region where the critical point is expected. The binodal is the lower curve in the phase diagram in Figure 1. In the inset a theoretically calculated phase diagram is given for comparison. The calculations are based on the theory given by Lekkerkerker and Stroobants. ${ }^{24}$ It should be mentioned that this theory is less reliable for the large polymer volume fractions that we are dealing with experimentally.

For static light scattering measurements, samples were prepared independently, for each of which the binodal was determined separately. Afterward the location of the binodal is scaled onto the previously determined binodal. This is done because the error in concentration of an independently prepared sample is about the same order as the difference in location of the binodal and spinodal: the experimental error in the colloid volume fraction of independently prepared samples is about $0.2 \%$ in $\phi_{\mathrm{c}}$, while the difference $\Delta \phi_{\mathrm{c}}$ between the binodal and the spinodal is also about $0.2 \%$.

B. Spinodal. 1. Static Light Scattering. To obtain the correlation length, the reciprocal scattered intensity is plotted against the square of the wave vector. An example of such a plot for various concentrations along a dilution line is given in Figure 2. The squared correlation length is equal to the ratio 


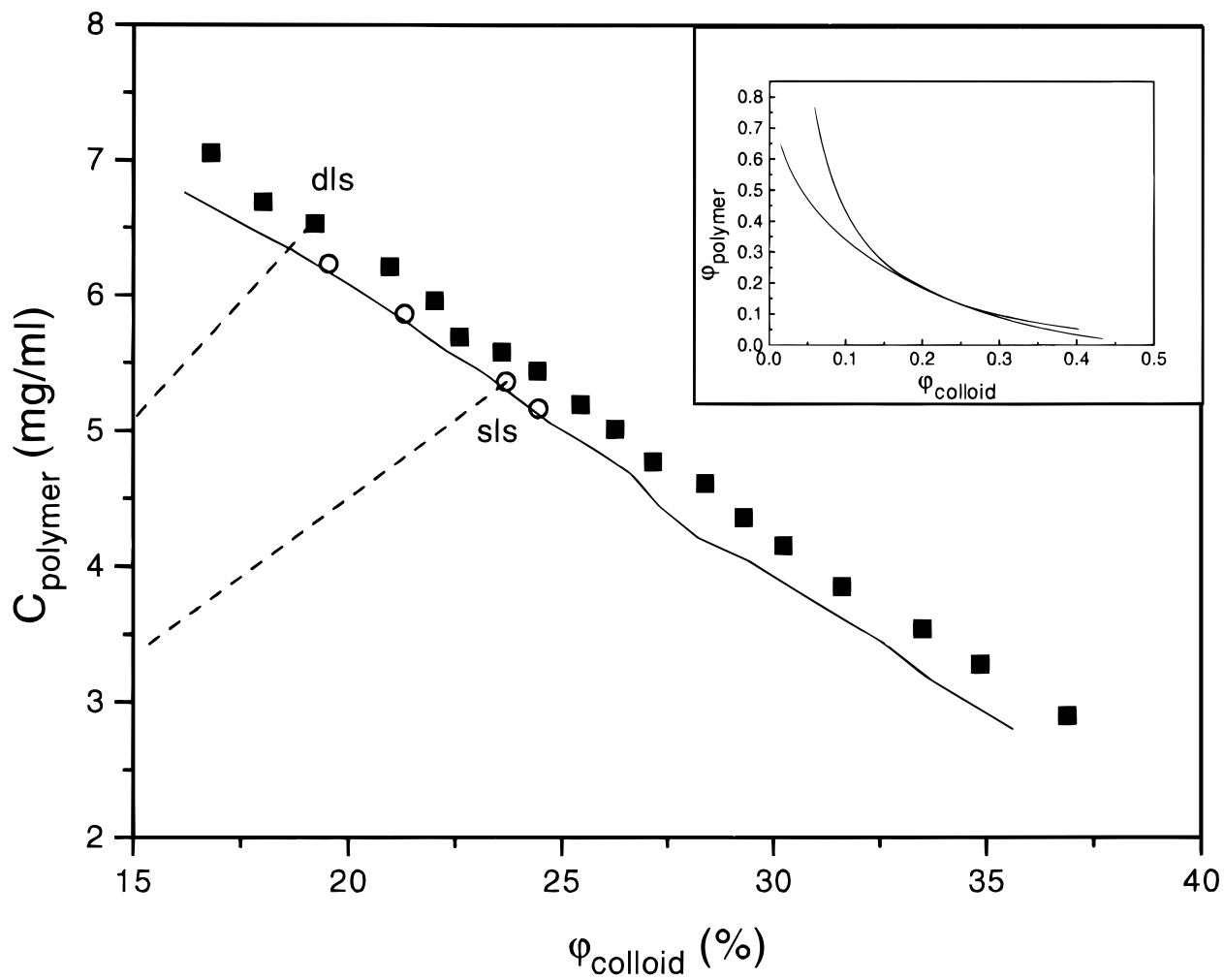

Figure 1. Experimental phase diagram of a colloid polymer mixture with a polymer/colloid size ratio of 0.48 . The system phase separates into a dilute and a dense disordered phase. The lower curve represents the binodal. The data points are spinodal points as determined from extrapolated static light scattering data and dynamic light scattering data. Two examples of dilution lines are given, viz., the lines that belong to Figures 2,3 and to Figures 5,6 . The errors in the static light scattering spinodal points are about $0.03 \%$, and in the dynamic light scattering spinodal points are about $0.3 \%$. The inset gives a theoretical phase diagram for comparison.

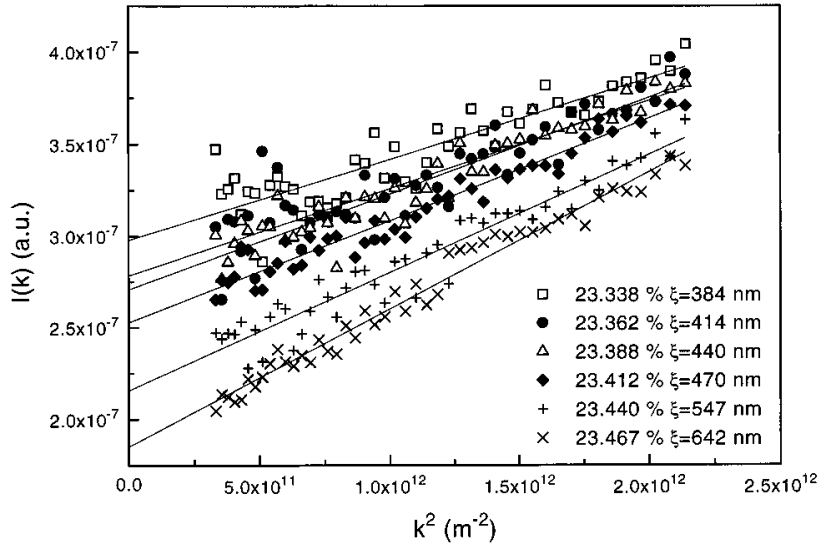

Figure 2. Example of an Ornstein-Zernike plot, where the reciprocal scattered intensity is plotted versus the square of the wave vector. The various curves relate to different concentrations along one dilution line. For the sake of clarity not all measurements are shown.

of the intercept and the slope. When plotting the inverse correlation length versus the volume fraction of colloid, a linear dependence is found as illustrated in Figure 3. This is in contrast with the critical exponent found for the divergence of the correlation length as a function of the temperature, which is $1 / 2$ in the mean field region and 0.63 beyond. ${ }^{11}$

A strong increase of the correlation length occurs within a small region of about $1 \%$ in $\phi_{c}$ below the binodal in the stable part of the phase diagram. The extrapolations to determine the location of the spinodal points are given in Figure 4. Note that the difference between the binodal and the spinodal is found to be in the range of $0.1 \%$. These spinodal points are depicted in Figure 1 with open circles. The error in determining the spinodal concentration with use of static light scattering is about $0.03 \%$.

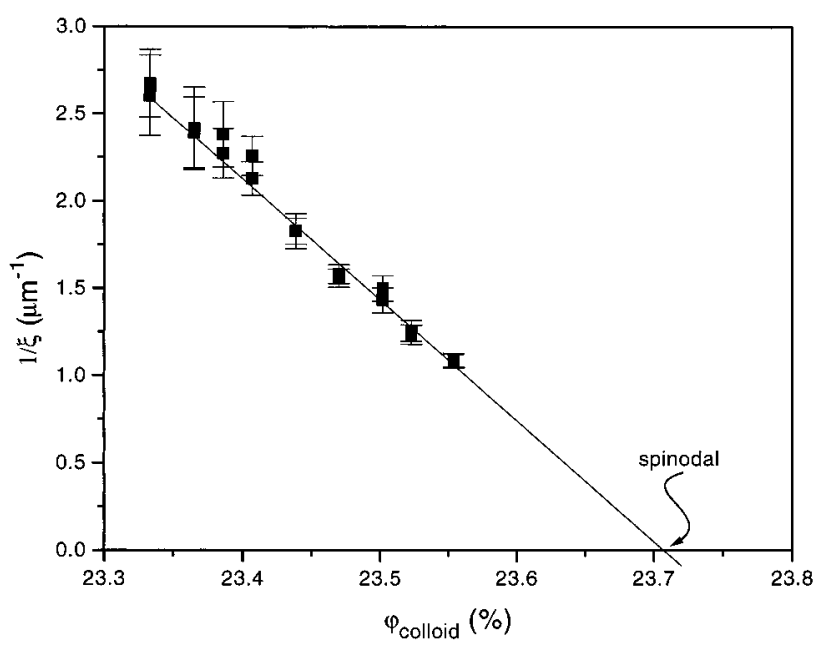

Figure 3. The inverse correlation length plotted versus the volume fraction of colloid, indicating a linear dependence. The data are from the same series of measurements as in Figure 2. The spinodal is at $\phi_{\mathrm{c}}=23.71 \pm 0.03 \%$.

2. Dynamic Light Scattering. The polymer solution itself scatters very little compared with the total scattered intensity, so this will be neglected in the analysis, and all the scattered intensity will be attributed to the colloidal particles.

The effective diffusion coefficient is obtained from the intensity autocorrelation function using a second cumulant fit, where the first cumulant is proportional to the diffusion coefficient.

In the region where the volume fraction of colloid at the binodal is smaller than $\phi_{\mathrm{c}} \approx 12 \%$, the dls measurements could not be analyzed according to the Ornstein-Zernike approximation: the diffusion coefficient was found to decrease with increasing wave vector, which would lead to an unphysical 


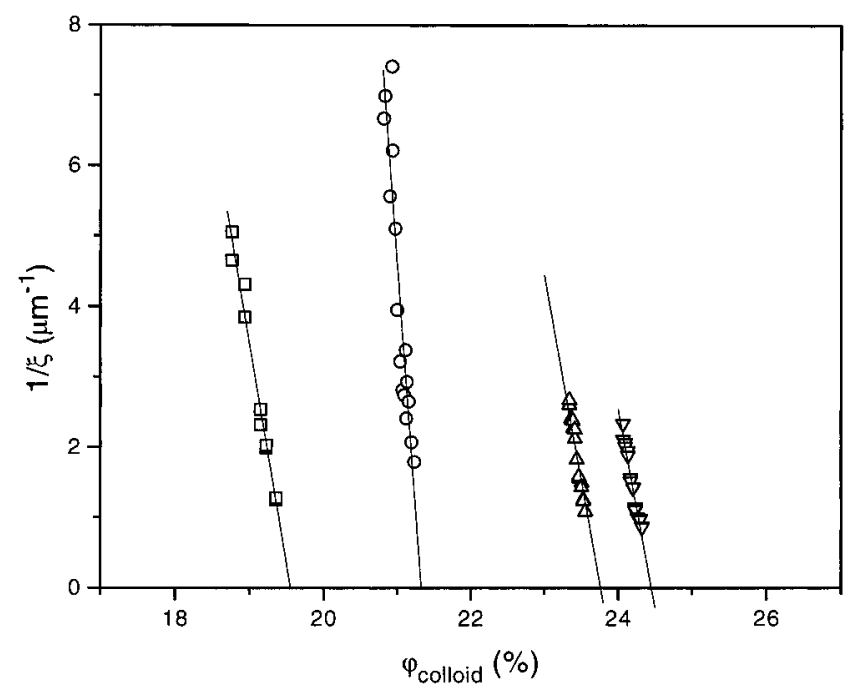

Figure 4. Inverse correlation length, as determined from static light scattering, plotted against $\phi_{\mathrm{c}}$. The solid curves are linear fits, which are used to determine the location of the spinodal points. The errors are of the same order as in Figure 3.

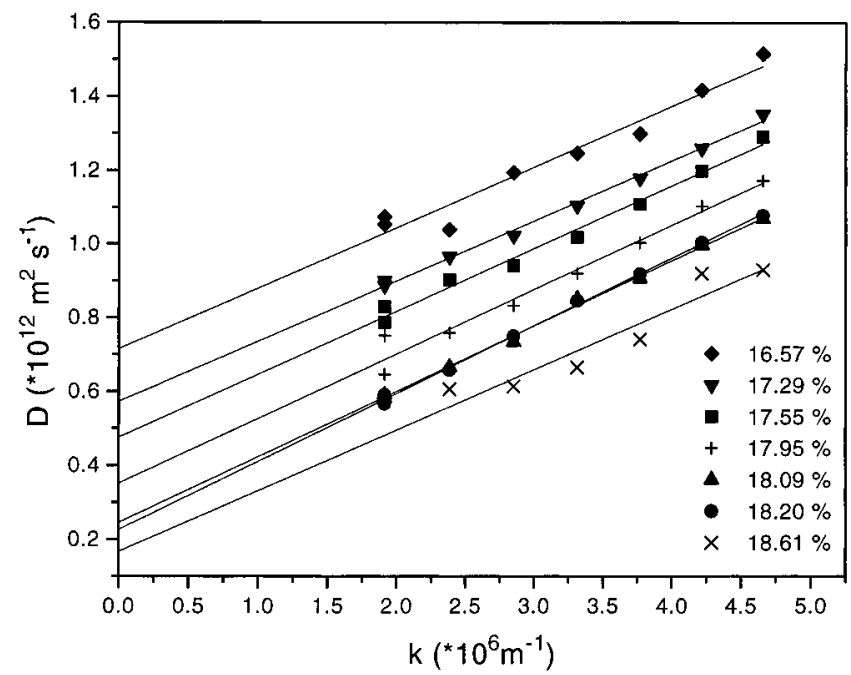

Figure 5. Example of a series of dynamic light scattering curves, where the diffusion coefficient is plotted versus the wave vector for different concentrations along one dilution line. For the sake of clarity not all measurements are shown.

negative correlation length. The spinodal in this part of the phase diagram is too far away from the binodal, so that attractions do not lead to the Ornstein-Zernike behavior.

As mentioned in the Theory section, linear extrapolation of $D$ versus $k$ to $k=0$ yields $D(k=0)=D_{0} \beta \Sigma / \xi^{2}$, and the slope of these curves is independent of the correlation length. An example of a set of $D$ versus $k$ curves along a dilution line is given in Figure 5. To within experimental error we indeed find a linear dependence of $D$ on $k$, and the $D(k=0)$ values decrease on approach of the spinodal. Extrapolation of $D(k=0)$ versus $\phi_{\mathrm{c}}$ to $D(k=0)=0$ results in a point in the phase diagram for the spinodal at that specific colloid/polymer concentration ratio. An example of such an extrapolation is given in Figure 6. The spinodal points found in this way are the upper filled squares in Figure 1. An accurate location of the critical point cannot be determined from these spinodal points.

An overview of all values of $D(k=0)$ and the extrapolations is given in Figure 7. From this figure it is also not directly clear where the critical point is situated, because there is no significant change in the diffusion coefficient measured at the binodal. It is remarkable that $D(k=0)$ remains relatively large,

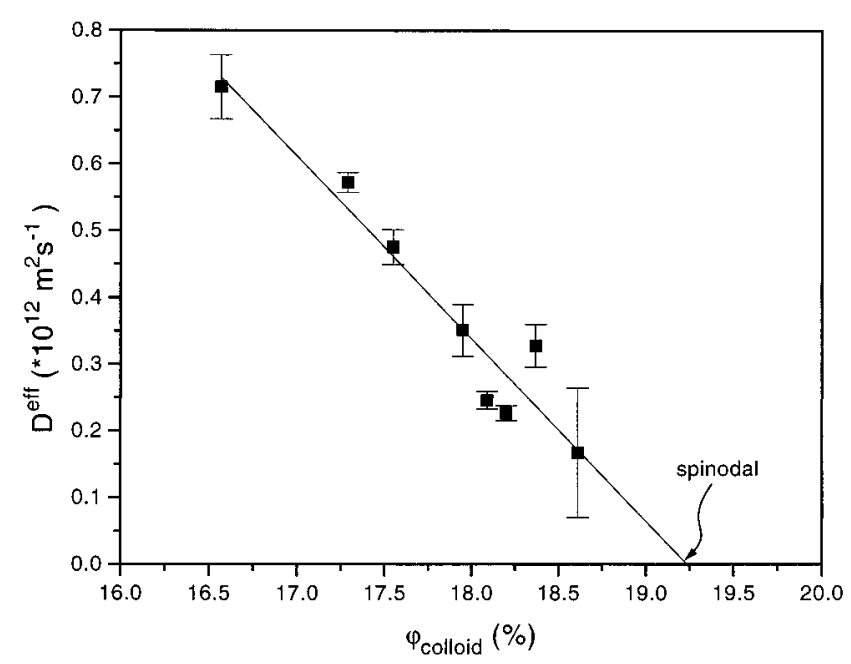

Figure 6. Example of an extrapolation of $D(k=0)$ versus $\phi_{c}$ to find a spinodal point. The data are the same as in Figure 5. The spinodal is at $\phi_{\mathrm{c}}=19.2 \pm 0.3 \%$.

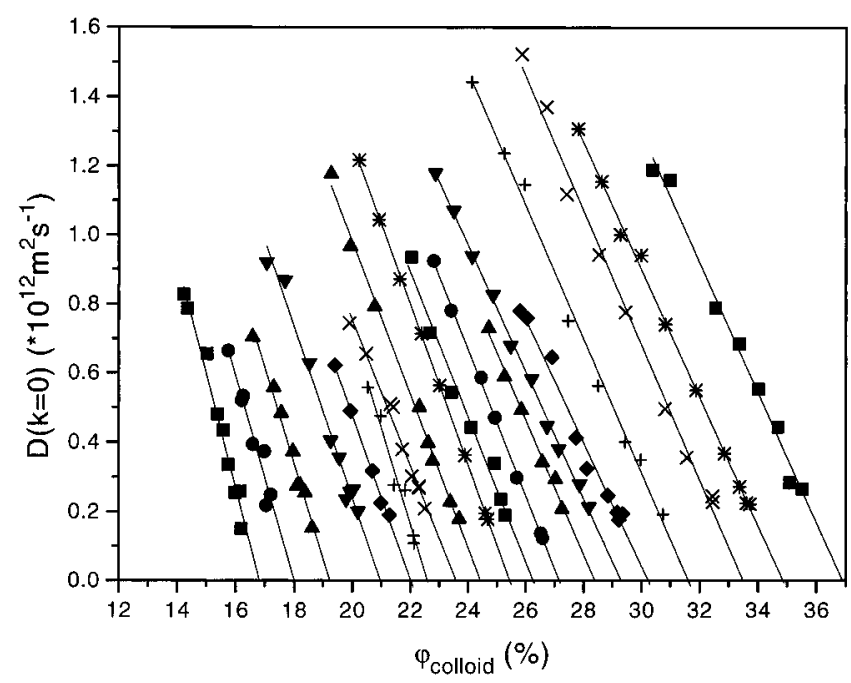

Figure 7. Zero wave vector diffusion coefficient plotted against the volume fraction of colloid for all the dilution lines measured. The solid curves are linear fits, which are used to determine the location of the spinodal points. The errors are of the same order as in Figure 6.

even in the region where the critical point is expected, based on visual observations.

It is expected that on the left as well as on the right of the critical point the distance between the binodal and spinodal increases when the distance from the critical point increases. This is not observed, although there is a single point that is closer to the binodal. It seems that the spinodal is almost parallel to the binodal. Compared with a theoretical phase diagram for a colloid polymer mixture with a size ratio of 0.5 , this is not too surprising, because the distance between the spinodal and binodal is indeed small in the entire region studied here. This renders the experimental determination of the exact location of the critical point problematic.

The fact that $D(k=0)$ is not very much enhanced at larger volume fractions may also be due to an increase of the solvent viscosity. In our case the solvent for the colloidal particles consists of a polymer solution. At first glance it seems that the viscosity of the solvent will decrease with increasing concentration ratio of colloid/polymer, because the binodal shifts to lower polymer concentration with increasing $\phi_{\mathrm{c}}$. However, this is an overall concentration, which has to be corrected for the fact that for increasing $\phi_{\mathrm{c}}$ the free volume available for the polymer decreases. In other words the colloidal particles 
exclude a part of the total volume for the polymer, so that the effective concentration of polymer increases, with an increasing colloid concentration. Using the following expression for the free volume ${ }^{6}$

$$
\alpha=\left(1-\phi_{c}\right) \mathrm{e}^{\left[-A \gamma-B \gamma^{2}-C \gamma^{3}\right]}
$$

in which $\gamma=\phi_{\mathrm{c}} /\left(1-\phi_{\mathrm{c}}\right), A=3 \sigma+3 \sigma^{2}+\sigma^{3}, B=9 \sigma^{2} / 2+$ $3 \sigma^{3}$, and $C=3 \sigma^{3}$ (where $\sigma$ denotes the size ratio $r_{\mathrm{p}} / r_{\mathrm{c}}$ ), it follows that the polymer concentration in the solvent at the binodal increases with a factor 2 , going from a $\phi_{\mathrm{c}}$ of $4 \%$ to $33 \%$. This increase of the polymer concentration gives rise to a lowering of $D_{0}$. However, correction of $D$ for this will not result in a different location for the spinodal from the extrapolations but will only result in an increasing value of $D(k=0)$ at the binodal. This increase will be larger at higher $\phi_{c}$. This explains why $D(k=0)$ is not very much enhanced at larger $\phi_{\mathrm{c}}$.

\section{Conclusions}

The binodal and spinodal are determined for a mixture of colloidal particles and polymer by means of visual observation and static and dynamic light scattering. It turns out that the distance between the spinodal and binodal is very small in the entire concentration range studied here, rendering the determination of the location of the critical point problematic. Compared with theory however, the agreement is quite good, as can be seen in Figure 1, except for absolute values of polymer concentrations. Notice that theoretically the binodal and spinodal are predicted to be very close together in a large region around the critical point.

On the basis of visual observations, where the volume ratio of the two separated phases is estimated, the critical point should be around $\phi_{\mathrm{c}}=21 \pm 2 \%$, which is in fairly good agreement with the theoretically calculated critical point. ${ }^{6}$ The experimental polymer concentrations are quite different from the theoretically predicted concentrations. When the volume fraction of polymer is calculated via number density and the radius of gyration, a volume fraction much larger than 1 is obtained. This seems physically impossible. However, these polymers can be regarded as coils that can interpenetrate. This explains the possibility of a volume fraction larger than 1 .

The critical exponent for the divergence of the correlation length is experimentally found to be 1 , in contrast with the mean field value of $1 / 2$ found for systems where the temperature is the relevant variable. The critical exponent is compared with the mean field value, because the spinodal is not approached sufficiently to suppose that this value can be compared with the 3D-Ising critical exponent of 0.63. From this, the conclusion can be drawn that the polymer concentration does not exactly play the role of an inverse temperature. This must be a result of a different dependence of the partition function on the polymer concentration than for conventional systems on the temperature. We do not know what the critical exponent theoretically should be in a colloid/polymer mixture.

The critical exponent found here pertains to the approach of the spinodal along dilution lines. Different exponents may be found on approach of the spinodal along different paths. Notice, however, that the various dilution lines show the same critical exponent.

There are two discrepancies to be noted between static and dynamic light scattering results. First of all, there is a difference between the location of the spinodal found with the two different techniques. Secondly, the extrapolated values of the diffusion coefficient, $D(k=0) \propto \xi^{-2}$, are found to be linear in the volume fraction. From this, one should expect that the correlation length is proportional to the square root of $\phi-\phi_{\mathrm{s}}$, in contrast with the linear dependence found with static light scattering. Both discrepancies are not yet understood.

Acknowledgment. We thank B. M. I. van der Zande for carrying out preliminary investigations on the dynamic light scattering measurements and A. Stroobants for calculating the theoretical phase diagram in Figure 1. This work was supported by the Stichting voor Fundamenteel Onderzoek der Materie (Foundation for Fundamental Research on Matter), which is part of the Nederlandse Organisatie voor Wetenschappelijk Onderzoek (Netherlands Organization for the Advancement of Research).

\section{References and Notes}

(1) Asakura, S.; Oosawa, F. J. Chem. Phys. 1954, 22, 1255.

(2) Vrij, A. Pure Appl. Chem. 1976, 48, 471.

(3) Gast, A. P.; Hall, C. K.; Russel, W. B. J. Colloid Interface Sci. 1983, 96,251

(4) Gas,t A. P.; Russel, W. B.; Hall, C. K. J. Colloid Interface Sci. 1986, 109, 161.

(5) Vincent, B.; Edwards, J.; Emmett, S.; Croot, R. Colloids Surf. 1988, $31,267$.

(6) Lekkerkerker, H. N. W.; Poon, W. C. K.; Pusey, P. N.; Stroobants, A.; Warren, P. B. Europhys. Lett. 1992, 20, 559.

(7) Poon, W. C. K.; Selfe, J. S.; Robertson, M. B.; Ilett, S. M.; Pirie, A. D.; Pusey, P. N. J. Phys. II 1993, 3, 1075. 653.

(8) Leal Calderon, F.; Bibette, J.; Biais, J. Europhys. Lett. 1993, 23,

(9) Pusey, P. N.; Pirie, A. D.; Poon, W. C. K. Physica A 1993, 201, 322.

(10) Pusey, P. N.; Poon, W. C. K.; Ilett, S. M.; Bartlett, P. J. Phys.: Condens.. Matter 1994, 6, A29.

(11) Stanley, H. Introduction to Phase Transitions and Critical Phenomena; Oxford University Press: New York, 1971.

(12) Grant, M. C.; Russel, W. B. Phys. Rev. E 1993, 47, 2606.

(13) Verduin, H.; Dhont, J. K. G. J. Colloid Interface Sci. 1995, 172, 425 .

(14) Dhont, J. K. G. An Introduction to Dynamics of Colloids; Elsevier Science B. V.: Amsterdam, 1996.

(15) Ackerson, B. J. J. Chem. Phys. 1976, 64, 242.

(16) Ackerson, B. J. J. Chem. Phys. 1978, 69, 684.

(17) Kawasaki, K. Ann. Phys. (NY) 1970, 61, 1.

(18) Ferrell, R. A. Phys. Rev. Lett. 1970, 24, 1169

(19) Fixman, M. Adv. Chem. Phys. 1964, 6, 175.

(20) Dhont, J. K. G.; Verduin, H. J. Chem. Phys. 1994 101, 6193. 62.

(21) Stöber, W.; Fink, A.; Bohn, E. J. Colloid Interface Sci. 1968, 26,

(22) van Helden, A. K.; Jansen, J. W.; Vrij, A. J. Colloid Interface Sci. 1981, 81, 354

(23) Philipse, A. P.; Vrij, A. J. Chem. Phys. 1987, 87, 5634.

(24) Lekkerkerker H. N. W.; Stroobants A. In Lecture Notes in Physics; Garrido, L. Ed.; Springer Verlag: Berlin, 1993; p 1.

JP962553V 